\title{
Effect of Lactobacillus rhamnosus GG on ileal pouch inflammation and microbial flora
}

Kuisma, J.

2003-02-15

Kuisma , J , Mentula , S , Järvinen , H , Kahri , A , Saxelin , M \& Färkkilä , M 2003 , ' Effect of Lactobacillus rhamnosus GG on ileal pouch inflammation and microbial flora ' , Alimentary Pharmacology \& Therapeutics , vol. 17 , no. 4 , pp. 509-515 . https://doi.org/10.1046/j.1365-2036.2003.01465.x

http://hdl.handle.net/10138/252763

https://doi.org/10.1046/j.1365-2036.2003.01465.x

publishedVersion

Downloaded from Helda, University of Helsinki institutional repository.

This is an electronic reprint of the original article.

This reprint may differ from the original in pagination and typographic detail.

Please cite the original version. 


\title{
Effect of Lactobacillus rhamnosus GG on ileal pouch inflammation and microbial flora
}

\author{
J. KUISMA*, S. MENTULA $\uparrow$, H. JARVINEN $\dagger$, A. KAHRI§, M. SAXELIN \& M. FARKKILA* \\ *Department of Gastroenterology, Helsinki University Central Hospital, †Department of Microbiology, National Public \\ Health Institute, †Department of Surgery, Helsinki University Central Hospital, §Department of Pathology, University of \\ Helsinki, and Valio Ltd, $R$ \& D, Helsinki, Finland \\ Accepted for publication 1 December 2002
}

\section{SUMMARY}

Background: Preliminary trials of probiotics in preventing recurrent chronic pouchitis have been encouraging. Aim: To investigate the efficacy of Lactobacillus GG supplementation as primary therapy for ileal pouch inflammation, and its effect on the microbial flora.

Methods: Twenty patients, with a previous history of pouchitis and endoscopic inflammation, were recruited for a prospective, randomized, double-blind, placebocontrolled trial of Lactobacillus GG supplementation (10 LGG, 10 placebo) in two gelatine capsules [(0.51) $\times 10^{10}$ colony-forming units/capsule] b.d. for 3 months. Quantitative bacterial culture of fresh faecal samples and biopsies taken from the pouch and afferent limb was performed before and after supplementation.
Results: Lactobacillus GG supplementation changed the pouch intestinal flora by increasing the ratio of total faecal lactobacilli to total faecal anaerobes $(P=0.03)$ and enhancing the frequency of lactobacilli-positive cultures in the pouch and afferent limb mucosal biopsy samples. However, only $40 \%$ of patients were colonized with Lactobacillus GG. No differences were observed between the groups with regard to the mean pouchitis disease activity index or the total anaerobes or aerobes of faecal or tissue biopsy samples.

Conclusions: A single-strain probiotic bacterium supplement of Lactobacillus GG changed the pouch intestinal bacterial flora, but was ineffective as primary therapy for a clinical or endoscopic response. More clinical trials are needed to evaluate the right placement and dosage of probiotics within a treatment regimen for pouchitis.

\section{INTRODUCTION}

The most significant late complication after restorative proctocolectomy and ileal pouch-anal anastomosis is pouchitis, which, depending on the diagnostic criteria used and the follow-up time after surgery, occurs in $20-59 \%$ of patients. ${ }^{1-3}$ Most episodes of pouchitis are single attacks usually responding to metronidazole or antibiotic combination therapy. ${ }^{4}$ The good response to antibiotic therapy directed against anaerobic bacteria suggests a role for the bacterial flora in the pouch.

Correspondence to: Dr J. Kuisma, Department of Internal Medicine, Hyvinkaa Hospital, Sairaalankatu 1, 05850 Hyvinkaa, Finland. E-mail: juha.kuisma@hus.fi
Probiotic bacteria are non-pathogenic micro-organisms belonging to the natural gastrointestinal flora that confer a health benefit by altering the indigenous microflora., 6 Lactobacilli, bifidobacteria and other members of the resident microflora are commonly selected as probiotics. They can influence intestinal physiology, either directly or indirectly through modulation of the endogenous ecosystem or immune system. ${ }^{7}$ Preliminary trials of probiotics in the maintenance treatment of chronic pouchitis have been encouraging. ${ }^{8}$ Lactobacillus rhamnosus GG is a safe probiotic bacterium, relatively resistant to acid and bile, which adheres in vitro to epithelial cells and intestinal mucus, ${ }^{9,10}$ elaborates an antimicrobial substance ${ }^{11}$ and transiently colonizes the human intestine. ${ }^{12,13}$ Lactobacillus GG has 
shown promise in the treatment of rotavirus, ${ }^{14}$ travellers' ${ }^{15}$ and relapsing Clostridium difficile ${ }^{16}$ diarrhoea.

Although recent studies have provided evidence that beneficial probiotic bacteria can reduce relapses of chronic pouchitis, many questions remain with regard to the dosage required and the selection of the probiotic strain: do we need probiotic cocktails together with antibiotics for a clinical response or is a single probiotic strain effective?

The aim of this study was to evaluate the clinical efficacy of a commercially available single-strain Lactobacillus GG preparation on ileal pouch-anal inflammation in patients with restorative proctocolectomy for ulcerative colitis. The colonization and influence of Lactobacillus GG on the pouch microbial flora were determined.

\section{MATERIALS AND METHODS}

\section{Subjects}

We analysed the medical histories of all ulcerative colitis patients ( $n=104$; male to female ratio, 53/51; mean age, 45.2 years; range, 23-70 years) who underwent restorative proctocolectomy with an ileal J pouch-anal anastomosis between 1985 and 1994 at the Department of Surgery, Helsinki University Central Hospital, and who had a control follow-up endoscopy between 1997 and 1999. Histories included clinical symptoms, endoscopic and histological findings in previous followup endoscopies and the follow-up time after ileal pouchanal anastomosis. The diagnosis of ulcerative colitis had been established in all subjects by standard endoscopic, histological and radiological methods, and by clinical assessment and proctocolectomy specimens.

Of the 104 patients, 44 had been treated once or several times for clinical symptoms indicative of pouchitis, and had endoscopic and histological inflammation in the latest follow-up endoscopy. Based on these criteria, all 44 subjects were recruited into a prospective, randomized, double-blind, placebo-controlled trial of Lactobacillus GG (Lactobacillus rhamnosus GG, ATCC53103) supplementation for 3 months. The exclusion criteria were as follows: (i) refusal to participate; (ii) antibiotic therapy within 1 month; (iii) active chronic pouchitis (intermittent or continuous medical treatment) or symptoms [fever, bleeding, increased number of stools (> 2/day within 1 week)] indicative of an acute exacerbation of pouchitis; (iv) pregnancy. A total of 22 study subjects were enrolled.
All patients gave written informed consent, and the study protocol was approved by the Ethics Committee of the hospital.

\section{Lactobacillus GG supplementation}

Subjects were randomized to receive treatment with gelatine capsules (Gefilus, Valio Ltd, Helsinki, Finland) containing Lactobacillus GG $\left[(0.5-1) \times 10^{10}\right.$ colonyforming units/capsule], with microcrystalline cellulose as a filling material, or microcrystalline cellulose only as a placebo. Each subject received four capsules of Lactobacillus GG (or placebo) per day as two equal doses for 3 months. Subjects were asked not to eat probiotic dairy products during the supplementation period.

\section{Clinical assessment}

All patients were examined by a single investigator (JK) for the recording of clinical symptoms (stool frequency/ $24 \mathrm{~h}$, abdominal cramps, rectal bleeding, fever) and medication.

Fresh faecal samples were collected prior to endoscopy. The pouch was emptied with water enemas. During flexible pouch endoscopy before biopsy, the mucosa was carefully rinsed with sterile water under direct visual control to remove all possible faecal contamination. Five separate tissue biopsy specimens (three for bacterial culture and two for histology) were obtained from the pouch and five from the afferent limb above the pouch. These specimens were taken from the regions exhibiting active inflammation endoscopically. Staples and suture lines were avoided. Tissue biopsy samples were thoroughly rinsed with downward jets of sterile saline and placed in a Stuart transport tube.

All histological biopsy specimens were reviewed in a blind fashion by one pathologist (AK). Serial sections of formalin-fixed, paraffin-embedded tissue were stained with haematoxylin and eosin. The haematoxylin and eosin-stained sections were scored on a scale of 0 (absent) to 3 (severe) for the degree of acute inflammation (polymorphonuclear leucocyte infiltration, ulcers) and for the degree of villous atrophy, according to the criteria of Moskowitz et al. ${ }^{17}$

\section{Bacteriological studies}

Bacteriological samples were immediately transported to the laboratory and processed within $2 \mathrm{~h}$. Specimens were 
weighed and ground in thioglycolate medium $(1: 10)$ in a tissue grinder. Faecal samples were homogenized, their pH measured (Benchtop 420 pH Meter, Orion, Beverly, MA, USA) and serially diluted $\left(10^{-1}-10^{-7}\right)$ in prereduced peptone-yeast extract broth ( $\mathrm{pH}$ 7.0). Appropriate dilutions and aliquots of the homogenates were plated on to several selective and non-selective agar media for the isolation of aerobic and anaerobic microbes. MRS (deMan Rogosa and Sharpe, Merck, Darmstadt, Germany) agar was used for the isolation of lactobacilli, Brucella agar supplemented with haemin and vitamin $\mathrm{K}_{1}$ for total counts of anaerobic bacteria, and blood agar (5\% sheep blood) for total counts of aerobic bacteria. MRS and Brucella plates were incubated in anaerobic jars filled with a gas mixture $\left(80 \% \mathrm{~N}_{2}, 10 \% \mathrm{CO}_{2}, 10 \% \mathrm{H}_{2}\right)$ and blood plates in a $7 \% \mathrm{CO}_{2}$ atmosphere, both at $37{ }^{\circ} \mathrm{C}$ for up to 14 days. Total counts of the main groups of aerobic and anaerobic bacteria and yeast were enumerated. Different colony morphotypes were recorded, enumerated and isolated for further study. Isolates were identified using established methods, including aerotolerance testing, Gram staining, biochemical tests and enzyme profiling. ${ }^{18,19}$ The species and clonal identity of selected Lactobacillus GG isolates were confirmed by an arbitrarily primed-PCR (AP-PCR) method. ${ }^{20}$

\section{Faecal bile acid composition}

Faecal bile acids were determined from a single stool sample by gas-liquid chromatography (HP 5890 series II, Hewlett-Packard, Wilmington, DE, USA) using a capillary column (HP-1, cross-linked methyl siloxane; length, $50 \mathrm{~m}$; diameter, $0.32 \mathrm{~mm}$; Hewlett-Packard, Wilmington, DE, USA). ${ }^{21}$ Results are given as the composition of individual bile acids expressed as a percentage of the total bile acids and micrograms of bile acids per gram of faeces.

\section{Statistical analyses}

All data are expressed as the mean \pm S.E.M., and statistical analyses were performed using NCSS 2000 software for Windows (NCSS statistical software, Kaysville, UT, USA). The primary measure of efficacy was the comparison of the pre-treatment and post-treatment pouchitis disease activity index (PDAI), with improvement defined as a reduction in the PDAI by at least three points. Comparisons between the study groups and statistical evaluations of the significance of the differences in the mean $\log _{10}$ bacterial counts obtained from faecal and intestinal samples were performed by the Mann-Whitney U-test. The Wilcoxon signed rank test was used to compare pre-treatment and post-treatment values. A comparison of incidence was performed using Fisher's exact test. A logistic regression analysis was performed to examine the effects of various independent factors on Lactobacillus GG colonization. A $P$ value of $<0.05$ was considered to be statistically significant.

\section{RESULTS}

Before treatment with Lactobacillus GG, all study subjects revealed signs of inflammation of the pouch mucosa, both endoscopically (oedema, granularity, friability, loss of vascular pattern, mucous exudate, ulcers) and histologically (polymorphonuclear leucocyte infiltration, ulcers). Two patients were excluded after the first endoscopy because of severe endoscopic pouchitis (large ulcers, stricture) requiring immediate antibiotic therapy. The clinical and demographic characteristics and disease history of the 20 study subjects are shown in Table 1. The administration of Lactobacillus GG was well tolerated, and none of the patients was withdrawn from the study because of side-effects.
Table 1. Demographic characteristics of the study subjects

\begin{tabular}{llll}
\hline & \multicolumn{2}{l}{ Treatment group } & \\
\cline { 2 - 3 } & $\begin{array}{l}\text { Placebo } \\
(n=10)\end{array}$ & $\begin{array}{l}\text { Lactobacillus GG } \\
(n=10)\end{array}$ & \\
\hline Sex (male/female) & $4 / 6$ & $7 / 3$ & 0.37 \\
Mean age (years) (range) & $48.2(34-61)$ & $46.4(31-70)$ & 0.30 \\
Body mass index (kg/m $\left.{ }^{2}\right)$ & $25.5 \pm 1.8$ & $25.6 \pm 1.6$ & 0.62 \\
Number of stools/24 h & $6.9 \pm 0.8$ & $7.6 \pm 1.0$ & 0.62 \\
Number of episodes of & $2.7 \pm 0.8$ & $3.4 \pm 1.0$ & 0.66 \\
$\quad$ pouchitis/follow-up & $1.9 \pm 0.2$ & $1.5 \pm 0.3$ & 0.30 \\
Degree of villous atrophy $(0-3)$ & & & \\
\hline
\end{tabular}

Values presented as number of patients or as means \pm S.E.M. 


\section{Clinical and microbiological results}

Table 2 shows the mean PDAI scores, faecal $\mathrm{pH}$ and bacterial counts in faecal and tissue biopsy samples obtained before and after treatment with Lactobacillus GG or placebo.

The baseline total PDAI score ranged from 5 to 13 in both study groups. One patient in the placebo group and two patients in the Lactobacillus GG group had baseline total PDAI scores below 7. Figure 1 shows the changes in total PDAI score for patients treated with Lactobacillus GG or placebo. No differences were found in the mean pre-treatment or post-treatment PDAI scores between the groups. Clinical response (reduction in PDAI score of $\geq 3$ ) occurred in one female patient. Her concentration of faecal Lactobacillus GG increased from 0 to $7 \log _{10}$ colony-forming units/g, and her PDAI score decreased from 7 to 3. After treatment, she felt subjectively better than before treatment.

No significant changes were seen in the concentrations of total anaerobic and aerobic bacteria in the faecal, pouch and afferent limb mucosal biopsy samples between the placebo and Lactobacillus GG groups before or after supplementation. There was a trend towards higher faecal concentrations of total lactobacilli (from $5.0 \pm 0.8$ to $6.9 \pm 0.5, P=0.08)$ and a higher prevalence of lactobacilli-positive cultures in the pouch and afferent limb mucosa in patients treated with Lactobacillus GG. The ratio of total lactobacilli to total anaerobes increased from $0.53 \pm 0.08$ to $0.81 \pm 0.1(P=0.03)$ in the Lactobacillus GG group (Table 2). No differences were observed in the total anaerobes, aerobes or total lactobacilli in the pouch or afferent limb mucosal biopsy samples.

\section{Colonization of the pouch with Lactobacillus GG}

None of the study subjects was Lactobacillus GG culturepositive before administration. During Lactobacillus GG administration, four of the 10 study subjects became faecal culture-positive for Lactobacillus GG. The effect of Lactobacillus GG supplementation on the counts of faecal and tissue biopsy bacteria in patients with and without faecal recovery of Lactobacillus GG is presented in Table 3. The mean faecal concentration of Lactobacillus GG-colonized patients was $6.11 \log _{10}$ colony-forming units/g (range, 2.5-8.7 $\log _{10}$ colony-forming units/g). They showed a trend towards a lower ratio of total

Table 2. Pouchitis disease activity index (PDAI) and bacterial counts ( $\log _{10}$ colony-forming units/g) in faecal and tissue biopsy samples before and after administration of Lactobacillus GG (LGG) or placebo in the study groups

\begin{tabular}{|c|c|c|c|c|c|c|}
\hline & \multicolumn{3}{|l|}{ Pre-treatment } & \multicolumn{3}{|l|}{ Post-treatment } \\
\hline & Placebo $(n=10)$ & LGG $(n=10)$ & $P$ & Placebo $(n=10)$ & LGG $(n=10)$ & $P$ \\
\hline PDAI (0-18) & $8.4 \pm 0.7$ & $8.0 \pm 0.8$ & 0.44 & $8.0 \pm 0.7$ & $8.0 \pm 1.1$ & 0.97 \\
\hline Faecal pH & $6.2 \pm 0.3$ & $6.2 \pm 0.1$ & 0.74 & $6.4 \pm 0.2$ & $6.4 \pm 0.3$ & 0.91 \\
\hline \multicolumn{7}{|l|}{ Faecal bacteria } \\
\hline Total anaerobes & $9.4 \pm 0.2$ & $9.5 \pm 0.2$ & 0.97 & $9.3 \pm 0.2$ & $9.1 \pm 0.6$ & 0.47 \\
\hline Total aerobes & $7.9 \pm 0.2$ & $8.1 \pm 0.2$ & 0.65 & $6.9 \pm 0.6$ & $7.9 \pm 0.2$ & 0.38 \\
\hline Total lactobacilli & $6.7 \pm 0.5$ & $5.0 \pm 0.8$ & 0.13 & $6.1 \pm 0.7$ & $6.9 \pm 0.5$ & 0.48 \\
\hline $\begin{array}{l}\text { Ratio total lactobacilli } \\
\text { to total anaerobes }\end{array}$ & $0.71 \pm 0.05$ & $0.53 \pm 0.08$ & 0.14 & $0.66 \pm 0.08$ & $0.81 \pm 0.1^{*}$ & 0.69 \\
\hline \multicolumn{7}{|l|}{ Pouch mucosa } \\
\hline Total anaerobes & $3.9 \pm 0.3$ & $4.0 \pm 0.4$ & 0.51 & $4.1 \pm 0.2$ & $4.2 \pm 0.3$ & 0.90 \\
\hline Total aerobes & $3.4 \pm 0.3$ & $3.5 \pm 0.2$ & 0.93 & $3.1 \pm 0.3$ & $3.1 \pm 0.3$ & 0.92 \\
\hline Total lactobacilli & $3.7 \pm 0.3$ & $3.2 \pm 0.4$ & 0.46 & $3.0 \pm 0.4$ & $3.2 \pm 0.3$ & 0.77 \\
\hline No. culture-positive & $4(40 \%)$ & $5(50 \%)$ & 0.65 & $5(50 \%)$ & $8(80 \%)$ & 0.35 \\
\hline \multicolumn{7}{|l|}{ Afferent limb } \\
\hline Total anaerobes & $4.2 \pm 0.2$ & $4.1 \pm 0.3$ & 0.91 & $3.0 \pm 0.3$ & $4.0 \pm 0.3$ & 0.03 \\
\hline Total aerobes & $3.0 \pm 0.3$ & $3.1 \pm 0.3$ & 0.77 & $2.5 \pm 0.2$ & $2.7 \pm 0.2$ & 0.56 \\
\hline Total lactobacilli & $2.3 \pm 0.3$ & $3.7 \pm 0.2$ & 0.04 & $2.6 \pm 0.3$ & $3.1 \pm 0.3$ & 0.25 \\
\hline No. culture-positive & $3(30 \%)$ & $5(50 \%)$ & 0.41 & $3(30 \%)$ & $7(70 \%)$ & 0.17 \\
\hline
\end{tabular}

Data expressed as mean \pm S.E.M. or as number of patients.

$P$ values are vs. placebo and were determined by the Mann-Whitney $U$-test or Fisher's exact test. $P<0.05$ was considered to be significant.

* Significant increase from baseline level $(P=0.03$, Wilcoxon signed rank test). 


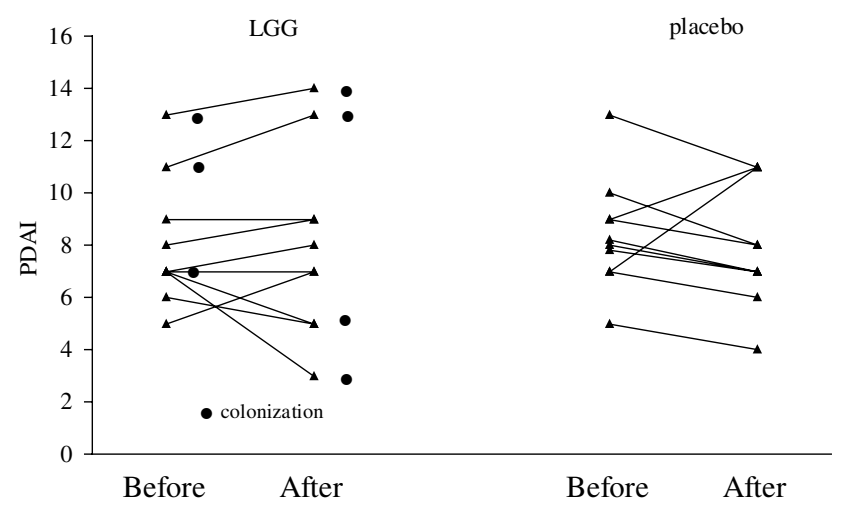

Figure 1. Pouchitis disease activity index (PDAI) before and after Lactobacillus GG (LGG) or placebo supplementation.

lactobacilli vs. faecal anaerobes $(0.37 \pm 0.02$ vs. $0.63 \pm 0.1, P=0.07)$ at baseline when compared with those without colonization. All biopsy samples from pouch and afferent limb mucosa were culture-negative for Lactobacillus GG. There was no significant difference in faecal primary (cholic acid, chenodeoxycholic acid) or secondary (deoxycholic acid, lithocholic acid) bile acid composition between placebo- and Lactobacillus GG-treated patients. Nor were any differences found in the mean pre-treatment faecal bile acid composition between those with and without colonization.
Possible factors influencing the colonization of the pouch with Lactobacillus GG were studied by logistic regression analysis, including pre-treatment PDAI, number of stools/day, time after surgery, faecal total primary bile acid (cholic acid + chenodeoxycholic acid) concentration, faecal $\mathrm{pH}$ and the number of exacerbations of pouchitis after surgery. No single factor was found to predict colonization.

\section{DISCUSSION AND CONCLUSIONS}

Lactobacillus GG, when administered twice daily in gelatine capsules for 3 months, was recovered in only $40 \%$ of study subjects. However, the supplementation increased the ratio of faecal total lactobacilli to total faecal anaerobes significantly, thus changing the intestinal flora in the ileo-anal pouches.

In our trial, no previous antibiotic medication was allowed, and none of the subjects was examined during acute exacerbation of pouchitis. Thus, we assumed that the baseline microbial and morphological changes represented a stable and long-term condition in the pouches. Based on these criteria, the most severe cases of pouchitis were excluded from the study. The mean PDAI score was therefore relatively low, ranging from 5 to 13 , before administration of the study preparations. However, if pouchitis is defined according to Sandborn

Table 3. Faecal and tissue biopsy counts of bacteria for Lactobacillus GG study group before and after administration

\begin{tabular}{|c|c|c|c|c|c|c|}
\hline & \multicolumn{3}{|l|}{ Pre-treatment } & \multicolumn{3}{|c|}{ Post-treatment } \\
\hline & \multicolumn{2}{|c|}{ Faecal recovery } & \multirow[b]{2}{*}{$P$} & \multicolumn{2}{|c|}{ Faecal recovery } & \multirow[b]{2}{*}{$P$} \\
\hline & Yes $(n=4)$ & No $(n=6)$ & & Yes & No & \\
\hline \multicolumn{7}{|l|}{ Faecal bacteria } \\
\hline Total anaerobes & $9.9 \pm 0.2$ & $9.3 \pm 0.2$ & 0.07 & $9.7 \pm 0.3$ & $8.7 \pm 0.9$ & 0.67 \\
\hline Total aerobes & $8.2 \pm 0.3$ & $8.1 \pm 0.4$ & 0.92 & $8.4 \pm 0.3$ & $7.5 \pm 0.3$ & 0.20 \\
\hline Total lactobacilli & $3.7 \pm 0.2$ & $5.8 \pm 1.1$ & 0.29 & $7.2 \pm 0.5$ & $6.7 \pm 0.8$ & 0.75 \\
\hline $\begin{array}{l}\text { Ratio total lactobacilli } \\
\text { to total anaerobes }\end{array}$ & $0.37 \pm 0.02$ & $0.63 \pm 0.1$ & 0.07 & $0.75 \pm 0.05$ & $0.86 \pm 0.2$ & 0.66 \\
\hline \multicolumn{7}{|l|}{ Pouch mucosa } \\
\hline Total anaerobes & $4.0 \pm 0.6$ & $4.0 \pm 0.5$ & 0.99 & $4.5 \pm 0.5$ & $3.9 \pm 0.3$ & 0.27 \\
\hline Total aerobes & $3.7 \pm 0.4$ & $3.3 \pm 0.3$ & 0.67 & $3.4 \pm 0.5$ & $2.9 \pm 0.2$ & 0.71 \\
\hline Total lactobacilli & $3.3 \pm 0.3$ & $3.2 \pm 0.6$ & 0.77 & $3.4 \pm 0.6$ & $3.1 \pm 0.4$ & 0.66 \\
\hline \multicolumn{7}{|l|}{ Afferent limb } \\
\hline Total anaerobes & $4.6 \pm 0.2$ & $3.8 \pm 0.5$ & 0.55 & $4.2 \pm 0.4$ & $3.7 \pm 0.5$ & 0.39 \\
\hline Total aerobes & $3.3 \pm 0.5$ & $3.0 \pm 0.4$ & 0.65 & $2.8 \pm 0.4$ & $2.7 \pm 0.2$ & 0.80 \\
\hline Total lactobacilli & $3.7 \pm 0.7$ & $3.6 \pm 0.3$ & 0.87 & $2.9 \pm 0.4$ & $3.5 \pm 0.3$ & 0.38 \\
\hline
\end{tabular}

Data expressed as mean \pm S.E.M. Bacterial count presented in $\log _{10}$ colony-forming units per gram of tissue.

Mann-Whitney $U$-test. $P<0.05$ was considered to be significant. 
et al. as a PDAI score of $\geq 7$ (range, $0-18),{ }^{22}$ nine of the 10 patients in the placebo group and eight of the 10 patients in the Lactobacillus GG group had pouchitis. Apart from one patient who felt that her condition improved, no marked difference in clinical, endoscopic or histological findings was found between baseline and the results after Lactobacillus GG supplementation. Thus, as primary therapy, Lactobacillus GG was not efficacious for the clinical improvement of pouch inflammation.

To treat patients with refractory pouchitis, Gionchetti et al. used a very high probiotic bacteria concentration (300 billion viable bacteria per gram) consisting of four strains of lactobacilli, three of bifidobacteria and one of Streptococcus salivarius ssp. thermophilus (VSL \#3, Yovis:Sigma-Tau, Pomezia, Italy). ${ }^{8}$ The 40 patients with chronic pouchitis who initially achieved remission after combination antibiotic therapy were randomized to receive placebo or VSL \#3 (3 g twice daily) for 9 months. In the probiotic-fed group, the relapse rate was significantly lower (15\%) than in the placebo group (100\%) after 9 months. In VSL \#3-treated patients, faecal concentrations of lactobacilli, bifidobacteria and Streptococcus salivarius increased significantly. The adhesion and colonization capacity of these strains on the ileal mucosa is unknown.

It is generally agreed that probiotic bacteria must be capable of survival and colonization of the intestinal tract before they can influence human health. ${ }^{23}$ The ability of Lactobacillus GG to survive in the intestinal canal $^{12,24}$ and to colonize the gastrointestinal tract $^{13,25}$ has been shown for both adults and children. ${ }^{26}$ In healthy intestines, the colonization is prolonged for about 1 week, ${ }^{25}$ but the colon of one patient with ulcerative colitis was not colonized by Lactobacillus GG. ${ }^{13}$ Our knowledge of the ability of Lactobacillus GG to colonize the human small bowel after colectomy is limited. In our trial, Lactobacillus GG was recovered in the faecal flora in $40 \%$ of cases, but was not found in mucosal biopsies. No single factor predicting survival was found in a logistic regression analysis. However, it is evident that the fast transit time, high stool frequency (mean of seven per day in our study), watery stool and changes in mucosal morphology (villous atrophy) may disturb the adherence and colonization of probiotic bacteria. As Lactobacillus GG is adherent to intestinal mucus, the bolus of bacteria ingested in capsules may be washed out, together with ileal secretions.

Pettersson et al., studying patients with an ileostomy, administered buttermilk and yoghurt (which contain viable lactobacilli) and pure cultures of $L$. bulgaricus or L. acidophilus. ${ }^{27}$ After feeding, they cultured the ileostomy effluent. Not even a transient increase in lactobacilli counts was found in the ileostomy contents in subjects receiving buttermilk, yoghurt and L. bulgaricus. Those receiving pure cultures of $L$. acidophilus, which is of human origin, showed transient increases in lactobacilli counts (1-3 logs) in the ileostomy effluent approximately $1.5 \mathrm{~h}$ after feeding, with a maximum at $3.5 \mathrm{~h}$; at $4.5 \mathrm{~h}$, however, over $99.5 \%$ of the administered organism had passed out of the small intestine. As a buffering material, milk protects bacteria. It is evident that higher doses of probiotics may be needed for their intestinal survival when administration is in a freezedried form.

The effects of probiotics can be direct, or indirect through the modulation of the endogenous flora or the immune system. ${ }^{28}$ The mechanisms of action of probiotic bacteria may be based on the modulation of cytokine expression, or on the induction of nitric oxide synthase and matrix metalloproteinase activity. ${ }^{29}$ Earlier studies have shown that Lactobacillus GG supplementation increases the faecal total lactobacilli anaerobic counts and bifidobacteria concentrations significantly. ${ }^{30,}{ }^{31}$ Madsen et al. have shown, in interleukin-10 gene-deficient mice, that normalization of colonic lumen Lactobacillus sp. levels reduces colonic mucosal adherent and translocated bacteria and prevents colitis. ${ }^{32}$ In the present study, no significant changes were noted in the concentrations of total anaerobes and aerobes in the faecal, pouch or afferent limb mucosa compared with the originally normal baseline levels. However, a significant increase occurred in the ratio of total lactobacilli to total anaerobes.

In conclusion, our data show that oral bacteriotherapy with the single-strain probiotic bacterium Lactobacillus GG can change the microbial flora in ileo-anal pouches. This study did not measure the effect of probiotic therapy on the recurrence of pouchitis. However, as primary therapy, Lactobacillus GG was inefficient for the clinical improvement of pouch inflammation. We recommend more placebo-controlled trials of probiotic use for pouchitis treatment.

\section{REFERENCES}

1 Simchuk EJ, Thirlby RC. Risk factors and true incidence of pouchitis in patients after ileal pouch-anal anastomosis. World J Surg 2000; 24: 851-6. 
2 Hurst R, Molinari M, Philip Chung TP, Rubin M, Michelassi F. Prospective study of the incidence, timing, and treatment of pouchitis in 104 consecutive patients after restorative proctocolectomy. Arch Surg 1996; 131: 497-502.

3 Meagher AP, Farouk R, Dozois RR, Kelly KA, Pemberton JH. J ileal pouch-anal anastomosis for chronic ulcerative colitis: complications and long-term outcome in 1310 patients. Br J Surg 1998; 85: 800-3.

4 Nasmyth DG, Godwin PG, Dixon MF, Williams NS, Johnton D. Ileal ecology after pouch-anal anastomosis or ileostomy. Gastroenterology 1989; 96: 817-24.

5 Bengmark S. Ecological control of the gastrointestinal tract. The role of probiotic flora. Gut 1998; 42: 2-7.

6 Fuller R. Probiotics in man and animal. J Appl Bacteriol 1989; 66: 365-78.

7 Marteau PR, de Vrese M, Cellier CJ, Schrezenmeir J. Protection from gastrointestinal diseases with the use of probiotics. Am J Clin Nutr 2001; 73: 430S-6S.

8 Gionchetti P, Rizzello F, Venturi A, et al. Oral bacteriotherapy as a maintenance treatment in patients with chronic pouchitis: a double-blind placebo-controlled trial. Gastroenterology 2000; 119: 305-9.

9 Tuomola EM, Salminen S. Adhesion of some probiotic and dairy Lactobacillus strains to Caco-2 cell cultures. Int J Food Microbiol 1998; 41: 45-51.

10 Ouwehand AC, Kirjavainen PV, Gronlund M-M, Isolauri E, Salminen SJ. Adhesion of probiotic micro-organisms to intestinal mucus. Int Dairy J 1999; 9: 623-30.

11 Silva M, Jacobs NV, Deneke C, et al. Antimicrobial substance from a human Lactobacillus strain. Antimicrob Agents Chemother 1987; 31: 1231-3.

12 Goldin BR, Gorbach SL, Saxelin ML, Barakat S, Gualtieri BS, Salminen S. Survival of Lactobacillus species (strain GG) in human gastrointestinal tract. Dig Dis Sci 1992; 37: 121-8.

13 Alander M, Korpela R, Saxelin M, Vilpponen-Salmela T, Mattila-Sandholm T, von Wright A. Recovery of Lactobcillus rhamnosus GG from human colonic biopsies. Lett Appl Microbiol 1997; 24: 361-4.

14 Isolauri E, Juntunen M, Rautanen T, Sillanaukee P, Koivula T. A human Lactobacillus strain GG (Lactobacillus casei sp. strain GG) promotes recovery from acute diarrhoea in children. Pediatrics 1991; 88: 90-7.

15 Hilton E, Kolakowski P, Smith M, Singer C. Efficacy of Lactobacillus GG as diarrheal preventive in travelers. J Travel Med 1997; 4: 41-3.

16 Bennet RG, Gorbach SL, Goldin BR, et al. Treatment of relapsing Clostridium difficile diarrhea with Lactobacillus GG. Nutrition Today 1996; 31(Suppl. 1): 35S-8S.

17 Moskowitz RL, Shephard NA, Nicholls RJ. An assessment of inflammation in the reservoir after restorative proctocolectomy with ileal reservoir. Int J Colorectal Dis 1986; 1: 167-74.

18 Summanen P, Baron EJ, Citron DM, Strong DM, Wexler HM, Finegold SM. Wadsworth Anaerobic Bacteriology Manual, 5th edn. Belmont, CA: Star Publishing, 1993.
19 Jousimies-Somer HR, Summanen PH, Finegold SM. Bacteroides, porphyromonas, prevotella, fucobacterium and other anaerobic gram-negative rods and cocci. In: Murray PR, Baron EJ, eds. Manual of Clinical Microbiology, 7th edn. Washington DC: ASM Press, 1999: 690-713.

20 Tynkkynen S, Satokari R, Saarela M, Mattila-Sandholm T, Saxelin M. Comparison of ribotyping, randomly amplified polymorphic DNA analyses, and pulse-field gel electrophoresis in typing of Lactobacillus rhamnosus and L. casei. Appl Environ Microbiol 1999; 65(9): 3908-14.

21 Grundy SM, Ahrens EH Jr, Miettinen TA. Quantitative isolation and gas-liquid chromatographic analysis of total fecal bile acids. J Lipid Res 1965; 6: 397-410.

22 Sandborn WJ, Tremaine WJ, Batss KP, Pemberton JH, Phillips SF. Pouchitis following ileal pouch-anal anastomosis: a pouchitis disease activity index. Mayo Clin Proc 1994; 69: 409-15.

23 Gorbach SL. Probiotics and gastrointestinal health. Am J Gastroenterol 2000; 95(Suppl.): S2-4.

24 Saxelin M, Ahokas M, Salminen S. Dose-response on the faecal colonization of Lactobacillus strain GG administered in two different formulations. Microb Ecol Health Dis 1993; 6: 119-22.

25 Alander M, Satokari R, Korpela R, et al. Persistence of colonization of human colonic mucosa by a probiotic strain, Lactobacillus rhamnosus GG, after oral consumption. Appl Environ Microbiol 1999; 65: 351-4.

26 Millar MR, Bacon C, Smith SL, Walker V, Hall MA. Enteral feeding of premature infants with Lactobacillus GG. Arch Dis Child 1993; 69: 483-7.

27 Pettersson L, Graf W, Sewelin U. Survival of Lactobacillus acidophilus NCDO 1748 in the human gastrointestinal tract. In: Hallegren B, ed. XV Symposium Swedish Nutrition Foundation. Uppsala: Almquist and Wilssell, 1983: 127-30.

28 Marteau P, Pochart P, Bouhnik Y, Rambaud JC. The fate and effects of transiting nonpathogenic microorganisms in the human intestine. World Rev Nutr Diet 1993; 74: 1-21.

29 Ulisse S, Gionchetti P, D'Alo S, et al. Expression of cytokines, inducible nitric oxide synthase, and matrix metalloproteinases in pouchitis: effects of probiotic treatment. Am J Gastroenterol 2001; 96: 2691-9.

30 Benno Y, He F, Hosada M, et al. Effects of Lactobacillus GG yogurt on human intestinal microecology in Japanese subjects. Nutrition Today 1996; 31(Suppl. 1): 9S-11S.

31 Apostolou E, Pelto L, Kirjavainen PV, Isolauri E, Salminen SJ, Gibson GR. Differences in the gut bacterial flora of healthy and milk-hypersensitive adults, as measured by fluorescence in situ hybridization. FEMS Immunol Med Microbiol 2001; 30: 217-21.

32 Madsen KL, Doyle JS, Jewell LD, Tavernini MM, Fedorak RN. Lactobacillus species prevent colitis in interleukin 10 genedeficient mice. Gastroenterology 1999; 116: 1107-14. 